\title{
On the solution of the purely nonlocal theory of elasticity as a limiting case of the two-phase theory
}

\author{
Gennadi Mikhasev ${ }^{\mathrm{a}}$, Andrea Nobilib,* \\ ${ }^{a}$ Department of Bio- and Nanomechanics, Belarusian State University, 4 Nezavisimosti \\ Ave., 220030 Minsk, Belarus \\ ${ }^{b}$ Engineering Department "Enzo Ferrari", Università degli Studi di Modena e Reggio \\ Emilia, 41125 Modena, Italy
}

\begin{abstract}
In the recent literature stance, purely nonlocal theory of elasticity is recognized to lead to ill-posed problems. Yet, we show that a meaningful energy bounded solution of the purely nonlocal theory may still be defined as the limit solution of the two-phase nonlocal theory. For this, we consider the problem of free vibrations of a flexural beam under the two-phase theory of nonlocal elasticity with an exponential kernel, in the presence of rotational inertia. After recasting the integro-differential governing equation and the boundary conditions into purely differential form, a singularly perturbed problem is met that is associated with a pair of end boundary layers. A multi-parametric asymptotic solution in terms of size-effect and local fraction is presented for the eigenfrequencies as well as for the eigenforms for a variety of boundary conditions. It is found that simply supported end conditions convey the weakest boundary layer and that, surprisingly, rotational inertia affects the eigenfrequencies only in the classical sense. Conversely, clamped and free conditions bring a strong boundary layer and eigenfrequencies are heavily affected by rotational inertia, even for the lowest mode, in a manner opposite to that brought by nonlocality. Remarkably, all asymptotic solutions admit a well defined and energy bounded limit as the local fraction vanishes and the purely nonlocal model is retrieved.
\end{abstract}

\footnotetext{
* Corresponding author

Email addresses: mikhasev@bsu.by (Gennadi Mikhasev), andrea.nobili@unimore.it (Andrea Nobili)
}

Preprint submitted to International Journal of Solids and Structures 
Therefore, we may define this limiting case as the proper solution of the purely nonlocal model. Finally, numerical results support the accuracy of the proposed asymptotic approach.

Keywords:

Two-phase nonlocal elasticity, Nonlocal theory of elasticity, Asymptotic method, Free vibrations

\section{Introduction}

The classical linear theory of elasticity suffers from the well known defect of not encompassing an internal length scale, which feature gives rise to selfsimilar predictions. Yet, any real material possesses an internal microstructure and some characteristic length thereof. Consequently, classical elasticity may be assumed as a suitable model inasmuch as the physical phenomena of interest occur at a scale much greater than the internal characteristic length of the material. Failure to meet this condition is effectively demonstrated by, for instance, the singular stress field at the tip of a crack and by the non-dispersive nature of wave propagation. Extensions of classical elasticity have been proposed, in the form of generalized continuum mechanics (GCM), in an attempt to remediate these shortfalls. An excellent historical overview of GCM, together with extensive bibliographic details, may be found in [17]. Among GCM theories, we mention the theory of micro-polar elasticity [2, 3, 25], the couple-stress and strain-gradient elasticity theories [35, 23] and the nonlocal theory of elasticity [7]. In particular, following [7], "linear theory of nonlocal elasticity, which has been proposed independently by various authors [...], incorporates important features of lattice dynamics and yet it contains classical elasticity in the long wave length limit". Nonlocal elasticity is based on the idea that the stress state at a point is a convolution over the whole body of an attenuation function (sometimes named kernel or nonlocal modulus) with the strain field [34]. Although several attenuation functions may be considered, they need to comply with some important properties which warrant that (a) classical elasticity is re- 
verted to in the limit of zero length scale and that (b) normalization is satisfied [6]. As an example, Helmholtz and bi-Helmoltz kernels have been widely used in 1-D problems, their name stemming from the differential operators they are Green's function of $[8,15]$. Since nonlocal elasticity naturally leads to integrodifferential equations whose solution is most often impractical, an equivalent differential nonlocal model (EDNM) was developed in [6]. In such form, nonlocal elasticity has been extensively applied to study elastodynamics of beams and shells as described in the recent review [4] and with special emphasis on the application to nanostructures [29]. Generally, EDNM leads to interesting mechanical effects, such as increased deflections and decreased buckling loads and natural frequencies (softening effect), when compared to classical elasticity. However, a number of pathological results have also emerged, which are often referred to as paradoxes $[16,10,15]$. For instance, for a cantilever beam under point loading, nonlocality brings no effect $[24,32,1]$. It should be remarked that many studies based on the EDNM employ boundary conditions in terms of macroscopic stresses, i.e. in classical form, and therefore they disregard the important effect of the boundary through nonlocality. Although this approach may be still adopted for long structures or in the case of localized deformations occurring away from the boundaries [20,21], it is generally inaccurate.

Very recently, Romano et al. [30] claimed that Eringen's purely nonlocal model (PNLM) leads to ill-posed problems for the differential form of the model is consistent inasmuch as an extra pair of boundary conditions, termed constitutive, is satisfied. In [5], a two-phase nonlocal model (TPNL) was introduced which combines, according to the theory of mixtures, purely nonlocal elasticity with classical elasticity, by means of the volume fractions $\xi_{1}$ and $\xi_{2}=1-\xi_{1}$. This model is immune from the inconsistencies of the PNLM and it has been adopted to solve the problem of static bending [33] and buckling [36] of EulerBernoulli (E-B) beams. Static axial deformation of a beam is considered in $[26,37]$, while semi-analytical solutions for the combined action of axial and flexural static loadings is given in [18]. Axial and flexural free vibrations of beams have also been considered in [19] and in [9]. In these works, either the 
TPNM is solved numerically or it is reduced, by adopting the solution presented in [28], to an equivalent higher-order purely differential model with a pair of extra boundary conditions. Despite this reduction, the differential model is still difficult to analyse, especially in the neighbourhood of the PNLM, that is for $\xi_{1}$ small. In this respect, we believe that the asymptotic approach may be put to great advantage in predicting the mechanical behaviour of nanoscale structures for a vanishingly small $\xi_{1}[36,19]$.

In this paper, we consider free vibrations of a flexural beam taking into account rotational inertia (Rayleigh beam), within the TPNM and having assumed the Helmholtz attenuation function. The integro-differential model is reduced to purely differential form with an extra pair of boundary conditions. Spotlight is set on developing asymptotic solutions valid for small microstructure and/or little local fraction. These solutions feature a pair of boundary layers located at the beam ends, whose strength depends on the constraining conditions. Numerical results support the accuracy of the expansions. Most remarkably, the asymptotic approach allows to investigate the behaviour of the solution in the neighbourhood of the PNLM, where the expansions are non-uniform. Nonetheless, they admit a perfectly meaningful, energy bounded limit, which may be taken as the solution of the PNLM. We point out that the existence of such limit has been observed numerically in [10] for free-free end conditions.

\section{Problem formulation}

\subsection{Governing equations}

For a flexural beam, vertical equilibrium gives

$$
\rho S \frac{\partial^{2} v}{\partial t^{2}}=\frac{\partial \hat{Q}}{\partial x}+q(x)
$$

while rotational equilibrium lends

$$
J \frac{\partial^{2} \varphi}{\partial t^{2}}=-\frac{\partial \hat{M}}{\partial x}+\hat{Q} .
$$

Here, $v=v(x, t)$ is the vertical displacement, $\hat{Q}$ and $\hat{M}$ are the dimensional shearing force and the bending moment, respectively, $\rho$ is the mass density, 
99

$J=\rho I$ is the mass second moment of inertia per unit length of the beam, that is proportional to the second moment of area $I, S$ is the cross-sectional area and $q(x)$ the vertical applied load. As well-known, it is $I=S r_{A}^{2}$, where $r_{A}$ is the radius of gyration. Assuming that the beam is homogeneous and that its cross-section is constant along the length, Eqs. $(1,2)$ give

$$
\frac{\partial^{2} \hat{M}}{\partial x^{2}}-\rho S \frac{\partial^{2} v}{\partial t^{2}}+J \frac{\partial^{4} v}{\partial x^{2} \partial t^{2}}+q=0
$$

that governs transverse vibrations of flexural beams accounting for rotational inertia. In the mixed nonlocal theory (MNLT) of elasticity, we have $[5,7]$

$$
\hat{M}=-E I\left(\xi_{1} \frac{\partial^{2} v}{\partial x^{2}}+\xi_{2} \int_{0}^{L} K(|x-\hat{x}|, \kappa) \frac{\partial^{2} w}{\partial \hat{x}^{2}} d \hat{x}\right),
$$

where $E I$ is the beam flexural rigidity, $L$ the beam length and $K(|x-\hat{x}|, \kappa)$ is the kernel or attenuation function. The kernel is positive, symmetric, and it rapidly decays away from $x$; the nonlocal parameter $\kappa=e_{0} a$ depends on the scale coefficient $e_{0}$ as well as on the internal length scale $a . \xi_{1}$ and $\xi_{2}$ take up the role of volume fractions and they represent, respectively, the local and the nonlocal phase ratios, such that $\xi_{1}+\xi_{2}=1$ and $\xi_{1} \xi_{2} \geq 0$. When $\xi_{1}=0$, Eq.(4) degenerates into the purely nonlocal model (PNLM), while, in contrast, the case $\xi_{1}=1$ corresponds to classical local elasticity.

In what follows, we consider the Helmholtz kernel

$$
K(|x-\hat{x}|, \kappa)=\frac{1}{2 \kappa} \exp \left(-\frac{|x-\hat{x}|}{\kappa}\right),
$$

97 which is frequently used for $1 \mathrm{D}$ problems [30]. We note that for the Helmholtz 98 kernel the following transformations are valid

$$
\frac{\mathrm{d}}{\mathrm{d} s} \int_{0}^{1} \mathrm{e}^{-\frac{|s-\hat{s}|}{\varepsilon}} y(\hat{s}) d \hat{s}=\frac{1}{\varepsilon}\left[\mathrm{e}^{\frac{s}{\varepsilon}} \int_{s}^{1} \mathrm{e}^{-\frac{\hat{s}}{\varepsilon}} y(\hat{s}) \mathrm{d} \hat{s}-\mathrm{e}^{-\frac{s}{\varepsilon}} \int_{0}^{s} \mathrm{e}^{\frac{\hat{s}}{\varepsilon}} y(\hat{s}) \mathrm{d} \hat{s}\right],
$$

and

$$
\frac{\mathrm{d}^{2}}{\mathrm{~d} s^{2}} \int_{0}^{1} \mathrm{e}^{-\frac{|s-\hat{s}|}{\varepsilon}} y(\hat{s}) \mathrm{d} \hat{s}=\frac{1}{\varepsilon^{2}} \int_{0}^{1} \mathrm{e}^{-\frac{|s-\hat{s}|}{\varepsilon}} y(\hat{s}) \mathrm{d} \hat{s}-\frac{2}{\varepsilon} y(s) .
$$


In particular, Eq.(7) corresponds to [30, Eq.(6)] and it may be rewritten as

$$
\int_{0}^{1}\left[\varepsilon^{2} \frac{\mathrm{d}^{2} K(|s-\hat{s}|, \varepsilon)}{\mathrm{d} s^{2}}-K(|s-\hat{s}|, \varepsilon)+\delta(|s-\hat{s}|)\right] y(\hat{s}) \mathrm{d} \hat{s}=0,
$$

whereupon $K(|s-\hat{s}|, \varepsilon)$ is the Green's function of the singularly perturbed operator $\mathbf{H}_{\varepsilon}=1-\varepsilon^{2} \frac{\mathrm{d}^{2}}{\mathrm{~d} s^{2}}$. It is trivial matter to prove impulsivity, i.e. $\lim _{\varepsilon \rightarrow 0} K(\mid s-$ $\hat{s} \mid, \varepsilon)=\delta(s-\hat{s})$, where $\delta(s)$ is Dirac's delta function. Furthermore, we observe that Eq.(6), evaluated at the beam ends $s=0,1$ and for $\xi=0$, lends the constitutive boundary conditions [30, Eq.(5)]

$$
\frac{\mathrm{d} M}{\mathrm{~d} s}(0)=\varepsilon^{-1} M(0), \quad \text { and } \quad \frac{\mathrm{d} M}{\mathrm{~d} s}(1)=-\varepsilon^{-1} M(1),
$$

where $M=L \hat{M} / E I$ is the dimensionless bending moment. Thus, the constitutive boundary conditions are really the expression, on the domain boundary, of a general feature of the solution that is related to the integral operator (4).

Introducing the dimensionless axial co-ordinate $s=x / L$, under the assumption of time-harmonic motion ( $i$ is the imaginary unit)

$$
v(s, t)=w(s) \exp (i \omega t),
$$

and upon multiplying throughout by $L^{4} / E I$, Eq.(3) may be turned in dimensionless form

$$
\xi_{1} \frac{\mathrm{d}^{4} w}{\mathrm{~d} s^{4}}+\left(\lambda^{4} \theta-\varepsilon^{-2} \xi_{2}\right) \frac{\mathrm{d}^{2} w}{\mathrm{~d} s^{2}}+\frac{\xi_{2}}{2 \varepsilon^{3}} \int_{0}^{1} \exp \left(-\frac{|\hat{s}-s|}{\varepsilon}\right) \frac{\mathrm{d}^{2} w(\hat{s})}{\mathrm{d} \hat{s}^{2}} \mathrm{~d} \hat{s}-\lambda^{4} w=0 .
$$

Here, use have been made of Eqs. $(4,5)$ and we have let the dimensionless ratios

$$
\theta=\frac{J}{\rho S L^{2}}=\left(\frac{r_{A}}{L}\right)^{2}, \quad \lambda^{4}=\frac{\rho S L^{4} \omega^{2}}{E I},
$$

together with the microstructure parameter

$$
\varepsilon=\frac{\kappa}{L} \ll 1 .
$$

Clearly, $\theta$ plays the role of an aspect ratio squared and $\varepsilon$ is a scale effect. Assuming $w \in C^{6}[0,1]$, twice differentiating Eq.(8), taking into account Eqs. $(6,7)$ and then subtracting, we get the governing equation in purely differential form

$$
\varepsilon^{2} \xi \frac{\mathrm{d}^{6} w}{\mathrm{~d} s^{6}}-\left(1-\varepsilon^{2} \theta \lambda^{4}\right) \frac{\mathrm{d}^{4} w}{\mathrm{~d} s^{4}}-\lambda^{4}\left(\varepsilon^{2}+\theta\right) \frac{\mathrm{d}^{2} w}{\mathrm{~d} s^{2}}+\lambda^{4} w=0,
$$

where, hereinafter, we adopt the shorthand $\xi=\xi_{1}$. Eq.(10) is a singularly perturbed ODE [14], with respect to the small parameter $\varepsilon \sqrt{\xi}$. 
Eq.(10) is supplemented by suitable boundary conditions (BCs) at the ends. For clamped ends (C-C conditions), we have two pairs of kinematical conditions

$$
\begin{aligned}
& w(0)=w^{\prime}(0)=0, \\
& w(1)=w^{\prime}(1)=0 .
\end{aligned}
$$

For simply supported (S-S) ends

$$
\begin{array}{ll}
w(0)=0, & M(0)=\xi w^{\prime \prime}(0)+M_{0}=0, \\
w(1)=0, & M(1)=\xi w^{\prime \prime}(1)+M_{1}=0,
\end{array}
$$

114 having let

$$
M_{0}=\frac{1-\xi}{2 \varepsilon} \int_{0}^{1} \mathrm{e}^{-\frac{\hat{s}}{\varepsilon}} w^{\prime \prime}(\hat{s}) \mathrm{d} \hat{s}, \quad M_{1}=\frac{1-\xi}{2 \varepsilon} \mathrm{e}^{-\frac{1}{\varepsilon}} \int_{0}^{1} \mathrm{e}^{\frac{\hat{s}}{\varepsilon}} w^{\prime \prime}(\hat{s}) \mathrm{d} \hat{s} .
$$

For free-free (F-F) ends, one has

$$
\begin{array}{ll}
M(0)=0, & Q(0)=\xi w^{\prime \prime \prime}(0)+\theta \lambda^{4} w^{\prime}(0)+\varepsilon^{-1} M_{0}=0 \\
M(1)=0, & Q(1)=\xi w^{\prime \prime \prime}(1)+\theta \lambda^{4} w^{\prime}(1)-\varepsilon^{-1} M_{1}=0 .
\end{array}
$$

The nonlocal end bending moments (13) may be rewritten in differential form with the help of the original integro-differential equation (8):

$$
\begin{aligned}
& M_{0}=-\varepsilon^{2} \xi w^{i v}(0)+\left[1-\xi-\varepsilon^{2} \theta \lambda^{4}\right] w^{\prime \prime}(0)+\varepsilon^{2} \lambda^{4} w(0), \\
& M_{1}=-\varepsilon^{2} \xi w^{i v}(1)+\left[1-\xi-\varepsilon^{2} \theta \lambda^{4}\right] w^{\prime \prime}(1)+\varepsilon^{2} \lambda^{4} w(1) .
\end{aligned}
$$

Consequently, the BCs may be recast in differential form as

$$
\begin{aligned}
& M(0)=w^{\prime \prime}(0)+\varepsilon^{2} N_{0}=0, \\
& M(1)=w^{\prime \prime}(1)+\varepsilon^{2} N_{1}=0, \\
& Q(0)=\xi w^{\prime \prime \prime}(0)+\theta \lambda^{4} w^{\prime}(1)+\varepsilon^{-1} M_{0}=0, \\
& Q(1)=\xi w^{\prime \prime \prime}(1)+\theta \lambda^{4} w^{\prime}(1)-\varepsilon^{-1} M_{1}=0,
\end{aligned}
$$


where, making use of the connections $(6,7)$, we have

$$
\begin{aligned}
& N_{0}=\varepsilon^{-2}\left(\xi_{2} w^{\prime \prime}(0)-M_{0}\right)=-\xi w^{i v}(0)-\theta \lambda^{4} w^{\prime \prime}(0)+\lambda^{4} w(0), \\
& N_{1}=\varepsilon^{-2}\left(\xi_{2} w^{\prime \prime}(1)-M_{1}\right)=-\xi w^{i v}(1)-\theta \lambda^{4} w^{\prime \prime}(1)+\lambda^{4} w(1) .
\end{aligned}
$$

Besides, to rule out spurious solutions which may have appeared owing to double differentiation, we introduce a pair of additional BCs. Indeed, evaluating at the beam ends the differential with respect to $s$ of the original governing equation (8), one arrives at

$$
\begin{aligned}
& \varepsilon^{3} \xi w^{v}(0)-\varepsilon^{2} \xi w^{i v}(0)-\left(1-\xi-\varepsilon^{2} \theta \lambda^{4}\right)\left[\varepsilon w^{\prime \prime \prime}(0)-w^{\prime \prime}(0)\right] \\
&-\varepsilon^{3} \lambda^{4} w^{\prime}(0)+\varepsilon^{2} \lambda^{4} w(0)=0, \\
& \varepsilon^{3} \xi w^{v}(1)+\varepsilon^{2} \xi w^{i v}(1)-\left(1-\xi-\varepsilon^{2} \theta \lambda^{4}\right)\left[\varepsilon w^{\prime \prime \prime}(1)+w^{\prime \prime}(1)\right] \\
&-\varepsilon^{3} \lambda^{4} w^{\prime}(1)-\varepsilon^{2} \lambda^{4} w(1)=0 .
\end{aligned}
$$

Dropping rotational inertia, the additional boundary conditions (18) coincide with the constitutive boundary conditions recently obtained by Fernández-Sáez and Zaera [9, Eqs.(59) and (60)], provided that we replace our $\varepsilon$ and $\lambda^{4}$ with their $h$ and $\lambda_{w}$, respectively. However, it should be remarked that in [9] the original integro-differential problem is reduced to the equivalent differential form extending to dynamics the original argument developed in [34] for statics. Such argument takes advantage of a result presented in [27], which really applies to inhomogeneous integral equations with a given right-hand side. In the case of dynamics, however, this right-hand side is a problem unknown, for it is really an acceleration term, and therefore the applicability of the reduction formula is questionable.

\section{Exact solution of the boundary-value problems}

The general solution of the ODE (10) is

$$
w(s)=\sum_{j=0}^{6} c_{j} \exp \left(b_{j} s\right),
$$


Besides, we have, at leading order,

$$
q=\frac{2}{27\left(\xi \varepsilon^{2}\right)^{3}}
$$

and $q>0$, whereupon out of the three real roots, two, say $Z_{3}<Z_{2}<0$, are negative and one, say $Z_{1}$, is positive. Upon reverting to the original variable $\zeta$, we see that $\zeta_{3}^{2}<0<\zeta_{2}^{2}<\zeta_{1}^{2}$. Indeed, we get the expansions (the sign is immaterial)

$$
\zeta_{1}=\frac{1}{\varepsilon \sqrt{\xi}}, \quad \zeta_{2}=\alpha, \quad \zeta_{3}=\imath \beta,
$$

with

$$
\begin{aligned}
& \alpha=\lambda_{0} \sqrt{-\frac{1}{2} \theta \lambda_{0}^{2}+\sqrt{1+\frac{\theta^{2} \lambda_{0}^{4}}{4}}}, \\
& \beta=\lambda_{0} \sqrt{\frac{1}{2} \theta \lambda_{0}^{2}+\sqrt{1+\frac{\theta^{2} \lambda_{0}^{4}}{4}}},
\end{aligned}
$$


whence $\zeta_{1,2}$ are convey an exponential solution, while $\zeta_{3}$ is related to an oscillatory solution. It is worth noticing that $\zeta_{1}$ blows up as $(\varepsilon \sqrt{\xi}) \rightarrow 0$, that is for a vanishingly small scale effect or in the purely nonlocal situation. Indeed, this very root accounts for the edge effect in this problem and it describes a boundary layer.

We observe that, in general, the frequency equation for the ODE (10), subject to suitable boundary conditions, appears in transcendental form

$$
F(\lambda ; \xi, \varepsilon)=0,
$$

wherein $\lambda$ is the sought-for eigenvalue. The numerical solution of this equation is not straightforward matter, especially for very small values of the local fraction $\xi$, see e.g. [9] and [34] where plots are given for $\xi>0.1$ and $\xi>0.05$, respectively. Indeed, when looking for the numerical roots of (19), we observe, after [31], that the transformation to canonical form lends a considerable numerical advantage over Cardano's formulas in that it provides purely real solutions. Conversely, Cardano's formulas are likely to introduce a very small spurious imaginary component, which is most likely the cause of the numerical difficulty encountered in the literature when dealing with small $\xi$. To estimate the eigenvalue $\lambda$ for any $\xi$ and, in particular, in the limiting case of the PNLM (that occurs as $\xi \rightarrow 0$ ), we consider an asymptotic expansion in the small parameter $\varepsilon$.

\section{Asymptotic solution of the boundary-value problems}

Following a standard asymptotic argument $[14,19]$ and similarly to the extraction of the edge effect in shells [11, 12], we seek a solution of the eigenvalue problem through superposition of a solution, $w^{(m)}$, valid in the interior of the beam (the so-called outer solution), with a pair of boundary layers, $w_{1}^{(e)}$ and $w_{2}^{(e)}$, fading off away from the left and from the right beam end, respectively,

$$
w(s, \varepsilon)=w^{(m)}(s)+\varepsilon^{\gamma_{1}} w_{1}^{(e)}(s, \varepsilon)+\varepsilon^{\gamma_{2}} w_{2}^{(e)}(s, \varepsilon),
$$


where

$$
\frac{\partial w^{(m)}}{\partial s} \sim w^{(m)}, \quad \frac{\partial w_{i}^{(e)}}{\partial s} \sim \varepsilon^{-\varsigma} w_{i}^{(e)} \quad \text { as } \quad \varepsilon \rightarrow 0 .
$$

The parameter $\varsigma$ is named the index of variation of the edge effect integrals, while $\gamma_{1}$ and $\gamma_{2}$ are the indices of intensity of the edge effect integrals near the left and right ends, respectively. The positive values of $\gamma_{i}$ depend on the boundary conditions and should be specified for each end.

\subsection{Boundary layer}

To derive an equation describing the beam behaviour in the vicinity of the ends (boundary layer), we zoom in by assuming $s=\varepsilon^{\varsigma} \sigma$ and $1-s=\varepsilon^{\varsigma} \sigma$, respectively for the left and for the right end. For either case, one obtains the distinguished limit $\varsigma=1$ and Eq. (10) is rewritten as

$$
\xi \frac{\mathrm{d}^{6} w_{i}^{(e)}}{\mathrm{d} \sigma^{6}}-\left(1-\varepsilon^{2} \theta \lambda^{4}\right) \frac{\mathrm{d}^{4} w_{i}^{(e)}}{\mathrm{d} \sigma^{4}}-\varepsilon^{2} \lambda^{4}\left(\theta+\varepsilon^{2}\right) \frac{\mathrm{d}^{2} w_{i}^{(e)}}{\mathrm{d} \sigma^{2}}+\varepsilon^{4} \lambda^{4} w_{i}^{(e)}=0,
$$

whose solution is sought in the form of an asymptotic series

$$
w_{i}^{(e)}=w_{i 0}^{(e)}+\varepsilon w_{i 1}^{(e)}+\varepsilon^{2} w_{i 2}^{(e)}+\ldots
$$

Substitution of (23) into (22) lends a sequence of differential equations in the unknowns $w_{i j}^{(e)}(\sigma), i=1,2 ; j=0,1,2, \ldots$. Here, we simply give the first two terms of the expansion in the original variable $s$

$$
\begin{aligned}
& w_{1}^{(e)}(s, \varepsilon)=a_{10} \mathrm{e}^{-\frac{s}{\varepsilon \sqrt{\xi}}}+\varepsilon \mathrm{e}^{-\frac{s}{\varepsilon \sqrt{\xi}}}\left[a_{11}+a_{10} \frac{\theta \lambda_{0}^{4}(1-\xi)}{2 \sqrt{\xi}} s\right]+O\left(\varepsilon^{2} \mathrm{e}^{-\frac{s}{\varepsilon \sqrt{\xi}}}\right), \\
& w_{2}^{(e)}(s, \varepsilon)=a_{20} \mathrm{e}^{-\frac{1-s}{\varepsilon \sqrt{\xi}}}+\varepsilon \mathrm{e}^{-\frac{1-s}{\varepsilon \sqrt{\xi}}}\left[a_{21}+a_{20} \frac{\theta \lambda_{0}^{4}(1-\xi)}{2 \sqrt{\xi}}(1-s)\right]+O\left(\varepsilon^{2} \mathrm{e}^{-\frac{1-s}{\varepsilon \sqrt{\xi}}}\right),
\end{aligned}
$$

172 where $a_{i j}(i=1,2 ; j=0,1,2, \ldots)$ are constants that will be determined in the following from the boundary conditions.

\subsection{The outer solution}

The displacement $w^{(m)}$ as well as the eigenvalue $\lambda$ are sought in the form of an asymptotic series

$$
\begin{gathered}
w^{(m)}=w_{0}+\varepsilon w_{1}+\varepsilon^{2} w_{2}+\ldots, \\
\lambda=\lambda_{0}+\varepsilon \lambda_{1}+\varepsilon^{2} \lambda_{2}+\ldots
\end{gathered}
$$


The leading term in the series corresponds to the solution of the classical local problem and $\lambda_{0}$ is the classical eigenvalue. Substituting (25) into the governing Eq.(10) and equating coefficients of like powers of $\varepsilon$ leads to the sequence of differential equations:

$$
\sum_{j=0}^{k} \mathbf{L}_{j} w_{k-j}=0, \quad k=0,1,2, \ldots
$$

where

$$
\begin{gathered}
\mathbf{L}_{0} z=\frac{\mathrm{d}^{4} z}{\mathrm{~d} s^{4}}+\theta \lambda_{0}^{4} \frac{\mathrm{d}^{2} z}{\mathrm{~d} s^{2}}-\lambda_{0}^{4} z, \quad \mathbf{L}_{1} z=-4 \lambda_{0}^{3} \lambda_{1} \mathbf{D} z, \quad \mathbf{D} z=z-\theta \frac{\mathrm{d}^{2} z}{\mathrm{~d} s^{2}}, \\
\mathbf{L}_{2} z=-\xi \frac{\mathrm{d}^{6} z}{\mathrm{~d} s^{6}}-\theta \lambda_{0}^{4} \frac{\mathrm{d}^{4} z}{\mathrm{~d} s^{4}}+\lambda_{0}^{4} \frac{\mathrm{d}^{2} z}{\mathrm{~d} s^{2}}-2 \lambda_{0}^{2}\left(3 \lambda_{1}^{2}+2 \lambda_{0} \lambda_{2}\right) \mathbf{D} z, \\
\mathbf{L}_{3} z=-4 \theta \lambda_{0}^{3} \lambda_{1} \frac{\mathrm{d}^{4} z}{\mathrm{~d} s^{4}}+4 \lambda_{0}^{3} \lambda_{1} \frac{\mathrm{d}^{2} z}{\mathrm{~d} s^{4}}-4 \lambda_{0}\left(\lambda_{0}^{2} \lambda_{3}+\lambda_{1}^{3}+2 \lambda_{0} \lambda_{1} \lambda_{2}\right) \mathbf{D} z, \ldots
\end{gathered}
$$

At leading order, one finds the homogeneous forth order ODE

$$
\mathbf{L}_{0} w_{0}=0
$$

whose general solution

$$
w_{0}(s)=c_{01} \sin (\beta s)+c_{02} \cos (\beta s)+c_{03} \mathrm{e}^{-\alpha s}+c_{04} \mathrm{e}^{\alpha(s-1)}
$$

depends on the constants, $c_{0 i}, i \in\{1,2,3,4\}$, to be determined through the boundary conditions. However, the ODE (27) is subject to six boundary conditions and the problem is to determine which of these correspond to the outer solution and which pertain to the boundary layer [14]. The procedure of splitting the boundary conditions also gives the indices of intensity of the boundary layer, $\gamma_{1}, \gamma_{2}$, as well as the constants $c_{0 k}, a_{i j}$. For this, one needs to insert the expansions $(21,24,25)$ into the boundary conditions and equate coefficients of like powers of $\varepsilon$, while imposing the following requirements:

- in the leading approximation, every end condition should be homogeneous and coincide with those of the classical local theory;

- the $k^{\text {th }}$-order approximation generates two equations coupling the constants $a_{i(k-1)}$ with the previous order approximation $w_{k-1}(s)$ evaluated at the boundaries. 


\subsection{Beam with simply supported ends}

Let both beam ends be simply supported (S-S conditions), as given by the boundary conditions (12) rewritten in differential form $(16 \mathrm{a}, 16 \mathrm{~b})$, together with the additional constraints (18). Substituting the expansions $(21,24,25)$ into these conditions, we determine the strength of either boundary layer $\gamma_{1}=\gamma_{2}=$ 3.

At leading order, we arrive at the homogeneous classical boundary conditions

$$
w_{0}(0)=w_{0}(1)=w_{0}^{\prime \prime}(0)=w_{0}^{\prime \prime}(1)=0,
$$

which give $c_{01}=C, c_{02}=c_{03}=c_{04}=0$ and the classical eigenforms

$$
w_{0}(s)=C \sin (\beta s), \quad \beta=\pi n, \quad n=1,2, \ldots
$$

In light of the definition (20b), we find the eigenfrequencies

$$
\lambda_{0}=\lambda_{0}^{(n)} \equiv \frac{\pi n}{\left[1+\theta(\pi n)^{2}\right]^{1 / 4}}, \quad n=1,2, \ldots,
$$

and, by (9), the corresponding dimensional frequencies $\omega_{0}=\sqrt{\frac{E I}{\rho S}}\left(\lambda_{0} / L\right)^{2}$.

Moving to first-order terms, we again obtain a set of homogeneous boundary conditions

$$
w_{1}(0)=w_{1}(1)=w_{1}^{\prime \prime}(0)=w_{1}^{\prime \prime}(1)=0,
$$

as well as formulas for the leading amplitude in the boundary layer (24):

$$
\begin{aligned}
& a_{10}=-\sqrt{\xi}(1-\sqrt{\xi}) w_{0}^{\prime \prime \prime}(0)=C \beta^{3} \sqrt{\xi}(1-\sqrt{\xi}), \\
& a_{20}=\sqrt{\xi}(1-\sqrt{\xi}) w_{0}^{\prime \prime \prime}(1)=C(-1)^{n+1} \beta^{3} \sqrt{\xi}(1-\sqrt{\xi}) .
\end{aligned}
$$

Consideration of the inhomogeneous ODE (26) arising in this approximation, alongside the associated homogeneous boundary conditions (31), yields the compatibility condition $\lambda_{1}=0$, whence

$$
w_{1}=C_{1} \sin (\beta s),
$$

where $C_{1}$ is an arbitrary constant. Without loss of generality, one can assume $w_{1} \equiv 0$, for this amounts to taking $C=C_{0}+\varepsilon C_{1}+\ldots$ 


$$
\mathbf{L}_{0} w_{2}=-\mathbf{L}_{2} w_{0} \equiv \xi \frac{\mathrm{d}^{6} w_{0}}{\mathrm{~d} s^{6}}+\theta \lambda_{0}^{4} \frac{\mathrm{d}^{4} w_{0}}{\mathrm{~d} s^{4}}-\lambda_{0}^{3}\left(\lambda_{0}+4 \theta \lambda_{2}\right) \frac{\mathrm{d}^{2} w_{0}}{\mathrm{~d} s^{2}}+4 \lambda_{0}^{3} \lambda_{2} w_{0}
$$

We thus arrive at the inhomogeneous BVP on "spectrum". Upon observing that the homogeneous boundary-value problem arising at leading order is selfconjugated and therefore possesses the solution $z(s)=w_{0}(s)$, we deduce the compatibility condition for the BVP $(33,34)$

$$
\int_{0}^{1} w_{0}(s) \mathbf{L}_{2} w_{0}(s) \mathrm{d} s=0,
$$

which readily gives the correction for the eigenvalue:

$$
\lambda_{2}=-\frac{\beta^{2}\left[\lambda_{0}^{4}\left(1+\theta \beta^{2}\right)-\xi \beta^{4}\right]}{4 \lambda_{0}^{3}\left(1+\theta \beta^{2}\right)} .
$$

On taking into account this result, Eq. (34) turns homogeneous and, without loss of generality, we can assume $w_{2} \equiv 0$.

Considering the third-order approximation, one obtains the inhomogeneous boundary conditions

$$
\begin{gathered}
w_{3}(0)=-a_{10}=-C \beta^{3} \sqrt{\xi}(1-\sqrt{\xi}), \\
w_{3}(1)=-a_{20}=C(-1)^{n} \beta^{3} \sqrt{\xi}(1-\sqrt{\xi}), \\
w_{3}^{\prime \prime}(0)=\theta \lambda_{0}^{4} a_{10}=C \theta \lambda_{0}^{4} \beta^{3} \sqrt{\xi}(1-\sqrt{\xi}), \\
w_{3}^{\prime \prime}(1)=\theta \lambda_{0}^{4} a_{20}=(-1)^{n+1} C \theta \lambda_{0}^{4} \beta^{3} \sqrt{\xi}(1-\sqrt{\xi})
\end{gathered}
$$

for the inhomogeneous ODE

$$
\mathbf{L}_{0} w_{3}=-\mathbf{L}_{3} w_{0} \equiv 4 \lambda_{0}^{3} \lambda_{3} \mathbf{D} w_{0}
$$


The compatibility condition for the boundary-value problem $(35,36)$ works out

$$
\begin{aligned}
& -w_{3}^{\prime \prime}(1) w_{0}^{\prime}(1)+w_{3}^{\prime \prime}(0) w_{0}^{\prime}(0)-w_{3}(1) w_{0}^{\prime \prime \prime}(1)+w_{3}(0) w_{0}^{\prime \prime \prime}(0) \\
& \quad+\theta \lambda_{0}^{4}\left[w_{3}(0) w_{0}^{\prime}(0)-w_{3}(1) w_{0}^{\prime}(1)\right]+4 \lambda_{0}^{3} \lambda_{3} \int_{0}^{1}\left(w_{0}-\theta w_{0}^{\prime \prime}\right) w_{0} \mathrm{~d} s=0,
\end{aligned}
$$

whence we get the next correction term for the eigenvalue

$$
\lambda_{3}=\frac{\beta^{6} \sqrt{\xi}(1-\sqrt{\xi})}{\lambda_{0}^{3}\left(1+\theta \beta^{2}\right)} .
$$

The eigenform correction $w_{3}$, satisfying the boundary conditions (35), is given by the sum of a particular solution $w_{3 p}$ of Eq.(36), with the homogenenous solution $w_{3 o}$. The former reads

$$
w_{3 p}(s)=C_{3 p} s \cos (\beta s),
$$

where

$$
C_{3 p}=2 C \lambda_{0}^{3} \lambda_{3} \frac{1+\theta \beta^{2}}{\beta\left(\alpha^{2}+\beta^{2}\right)}=2 C \frac{\beta^{5}}{\alpha^{2}+\beta^{2}} \sqrt{\xi}(1-\sqrt{\xi}) .
$$

Consequently, making use of (37), we get

$$
\begin{aligned}
w_{3}(s) & =C \beta^{3} \sqrt{\xi}(1-\sqrt{\xi})\left\{c_{32} \cos (\beta s)+c_{33} \exp (-\alpha s)\right. \\
& \left.+c_{34} \exp [\alpha(s-1)]-2 c_{32} s \cos (\beta s)\right\},
\end{aligned}
$$

with the constants

$$
\begin{gathered}
c_{32}=-\beta^{2} /\left(\alpha^{2}+\beta^{2}\right), \\
c_{33}=\frac{1}{2} \alpha^{2} \mathrm{e}^{\alpha}(1-\operatorname{coth} \alpha)\left[\mathrm{e}^{\alpha}+(-1)^{n}\right] /\left(\alpha^{2}+\beta^{2}\right), \\
c_{34}=-\frac{1}{2} \alpha^{2} \mathrm{e}^{\alpha}(1-\operatorname{coth} \alpha)\left[(-1)^{n} \mathrm{e}^{\alpha}+1\right] /\left(\alpha^{2}+\beta^{2}\right) .
\end{gathered}
$$

Breaking at this step the asymptotic procedure for seeking the eigenvalues $\lambda_{k}$ and the associated eigenfunctions $w_{k}$, we obtain the asymptotic expansion

$$
\lambda=\lambda_{0}\left[1-\frac{1}{4} \varepsilon^{2} \beta^{2}(1-\xi)+\varepsilon^{3} \beta^{2} \sqrt{\xi}(1-\sqrt{\xi})+O\left(\varepsilon^{4}\right)\right],
$$



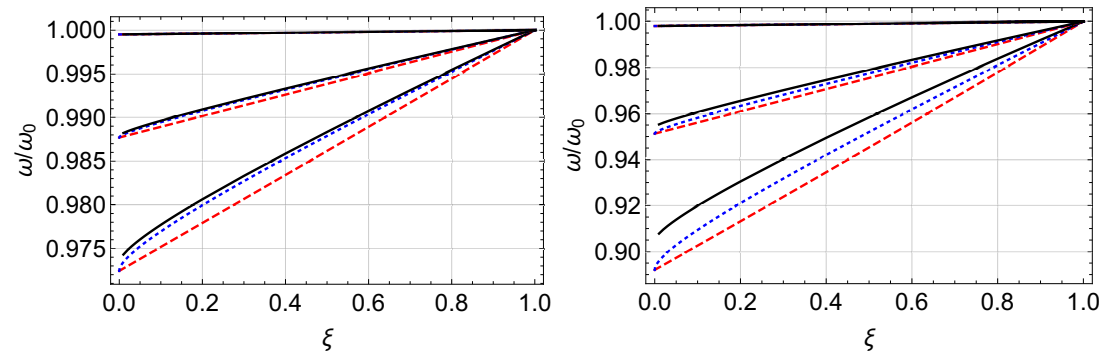

Figure 1: 1st (left) and 2nd (right) eigenfrequencies $\omega$ for a S-S beam (solid, black), with $\varepsilon=0.01,0.05$ and 0.075 , superposed onto the 1-term (dashed, red) and the 2-term (dotted, blue) asymptotic approximation, normalized with respect to the classical local frequency $\omega_{0}$, Eq.(39)

where $\beta$ and $\lambda_{0}$ are determined by (29) and (30), respectively. Up to an undetermined factor, the associated eigenmode reads

$$
\begin{gathered}
w(s)=\sin (\pi n s)+\varepsilon^{3}(\pi n)^{3} \sqrt{\xi}(1-\sqrt{\xi})\left\{c_{32} \cos (\pi n s)+c_{33} \exp (-\alpha s)\right. \\
+c_{34} \exp [\alpha(s-1)]-2 c_{32} s \cos (\pi n s) \\
\left.+\exp \left(-\frac{s}{\varepsilon \sqrt{\xi}}\right)+(-1)^{n+1} \exp \left(\frac{s-1}{\varepsilon \sqrt{\xi}}\right)\right\}+O\left(\varepsilon^{4}\right) .
\end{gathered}
$$

It is of interest to compare the dimensional natural frequency, $\omega$, determined with the TPNM, with its classical counterpart, $\omega_{0}$, evaluated within the framework of local elasticity, i.e. for $\xi=1$. When taking into account Eq.(9), we arrive at the relation

$$
\frac{\omega}{\omega_{0}}=\left(\lambda / \lambda_{0}\right)^{2}=1-\frac{1}{2} \varepsilon^{2}(\pi n)^{2}(1-\xi)+2 \varepsilon^{3}(\pi n)^{2} \sqrt{\xi}(1-\sqrt{\xi})+O\left(\varepsilon^{4}\right) .
$$

Remarkably, this expression is independent of $\theta$ and this unexpected feature is indeed confirmed by the numerical solution of the TPNM, see Fig.5. Fig.1 plots the approximation (39) in the range $0<\xi<1$ againts the numerical solution of the TPNM (given for $\xi>0.01$ ) for the scale parameter $\varepsilon=0.01,0.05$ and 0.075 . It appears that the 1-term asymptotic approximation is remarkably effective for small values of $\varepsilon$. The numerical solution of the TPNM given in Fig.1 compares favourably with the corresponding solution depicted in Fig.4 of [10] that, however, pertains to the range $\xi_{1}>0.1$, presumably owing to the 
as well as the frequency equation

$$
\frac{1}{2} \theta \lambda_{0}^{2} \sin \beta \sinh \alpha+\cos \beta \cosh \alpha-1=0 .
$$

In particular, if $\theta=0$, one arrives at the classical frequency equation, $\cosh \lambda_{0} \cos \lambda_{0}=$ 1, valid for a Bernoulli-Euler beam that disregards the rotational inertia of the cross-section, the corresponding eigenmode being

$$
w_{0}(s)=C\left[U\left(\lambda_{0} s\right)-\frac{U\left(\lambda_{0}\right)}{V\left(\lambda_{0}\right)} V\left(\lambda_{0} s\right)\right],
$$

where $S(x), T(x), U(x), V(x)$ are the well-known Krylov-Duncan functions [13, $\S 14.4 .3]$

$$
\begin{aligned}
S(x) & =\frac{1}{2}(\cosh x+\cos x), & T(x) & =\frac{1}{2}(\sinh x+\sin x), \\
U(x) & =\frac{1}{2}(\cosh x-\cos x), & V(x) & =\frac{1}{2}(\sinh x-\sin x) .
\end{aligned}
$$


Besides, we get

$$
\begin{aligned}
& a_{10}=\sqrt{\xi}(1-\sqrt{\xi}) w_{0}^{\prime \prime}(0), \\
& a_{20}=\sqrt{\xi}(1-\sqrt{\xi}) w_{0}^{\prime \prime}(1) .
\end{aligned}
$$

In the first-order approximation, one has the inhomogeneous ODE (26)

$$
\mathbf{L}_{0} w_{1}=4 \lambda_{0}^{3} \lambda_{1} \mathbf{D} w_{0}
$$

and the procedure of splitting the boundary conditions gives

$$
\begin{gathered}
w_{1}(0)=w_{1}(1)=0, \\
w_{1}^{\prime}(0)=(1-\sqrt{\xi}) w_{0}^{\prime \prime}(0), \quad w_{1}^{\prime}(1)=-(1-\sqrt{\xi}) w_{0}^{\prime \prime}(1) .
\end{gathered}
$$

The compatibility conditions for the BVP $(44,45)$ reads

$$
\begin{aligned}
w_{1}^{\prime}(1) w_{0}^{\prime \prime}(1)-w_{1}^{\prime}(0) w_{0}^{\prime \prime}(0)-w_{1}(1) w_{0}^{\prime \prime \prime}(1) & +w_{1}(0) w_{0}^{\prime \prime \prime}(0) \\
& -4 \lambda_{0}^{3} \lambda_{1} \int_{0}^{1} \mathbf{D} w_{0}(s) w_{0}(s) \mathrm{d} s=0,
\end{aligned}
$$

${ }^{43}$ whence, accounting for Eqs.(45), one obtains the correction

$$
\lambda_{1}=-\lambda_{0} \frac{(1-\sqrt{\xi})\left\{\left[w_{0}^{\prime \prime}(0)\right]^{2}+\left[w_{0}^{\prime \prime}(1)\right]^{2}\right\}}{4 \int_{0}^{1}\left[w_{0}^{\prime \prime}(s)\right]^{2} \mathrm{~d} s},
$$

where part-integration has been used at the denominator. Now, we can write the problem solution

$$
w_{1}(s)=c_{11} \sin (\beta s)+c_{12} \cos (\beta s)+c_{13} e^{-\alpha s}+c_{14} e^{\alpha(s-1)}+w_{1 p}(s),
$$

where

$$
\begin{aligned}
w_{1 p}(s)= & 2 \frac{\lambda_{0}^{3} \lambda_{1}}{\alpha^{2}+\beta^{2}} s\left\{\frac{1+\theta \beta^{2}}{\beta}\left[-c_{01} \cos (\beta s)+c_{02} \sin (\beta s)\right]\right. \\
& \left.+\frac{1-\theta \alpha^{2}}{\alpha}\left[c_{03} \mathrm{e}^{-\alpha s}-c_{04} \mathrm{e}^{\alpha(s-1)}\right]\right\}
\end{aligned}
$$

is the particular solution of Eq.(44) with the coefficients $c_{0 j}$ being given by Eqs.(41). In the special case of no rotational inertia, $\theta=0$, Eq.(46) may be reduced to the very simple expression

$$
\lambda_{1}=-2 \lambda_{0}(1-\sqrt{\xi}),
$$



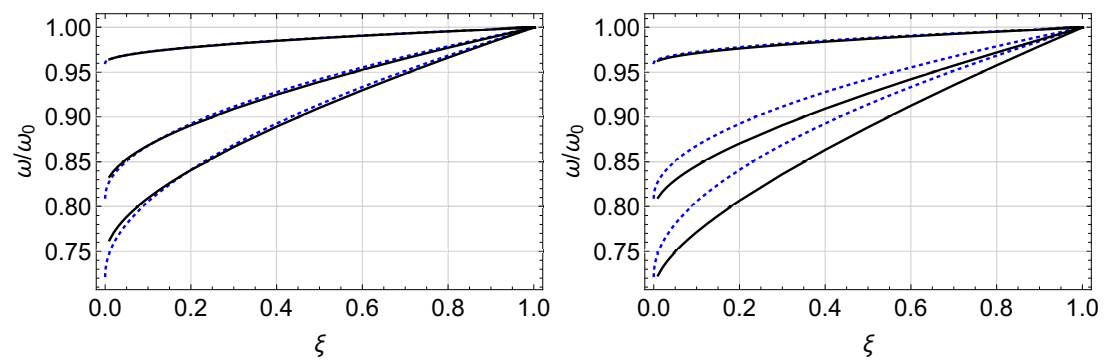

Figure 2: 1st (left) and 2nd (right) eigenfrequencies $\omega$ for a C-C beam (solid, black) in the absence of rotatory inertia, $\theta=0$, and with $\varepsilon=0.01,0.05$ and 0.075 , superposed onto the 1-term (dotted, blue) asymptotic approximation, normalized with respect to the classical local frequency $\omega_{0}$, Eq.(50)

and Eq.(48) gives

$$
w_{1 p}(s)=\frac{\lambda_{1}}{\lambda_{0}} s w_{0}^{\prime}(s)=-2 C(1-\sqrt{\xi}) \lambda_{0} s\left[T\left(\lambda_{0} s\right)-\frac{U\left(\lambda_{0}\right)}{V\left(\lambda_{0}\right)} U\left(\lambda_{0} s\right)\right] .
$$

Similarly, Eq.(47) becomes

$$
w_{1}(s)=C(1-\sqrt{\xi}) \lambda_{0}\left[T\left(\lambda_{0} s\right)-\frac{T\left(\lambda_{0}\right)}{V\left(\lambda_{0}\right)} V\left(\lambda_{0} s\right)\right]+w_{1 p}(s) .
$$

Breaking the asymptotic procedure at this step, we can write down the approximate formula for the nonlocal-to-local frequency ratio

$$
\frac{\omega}{\omega_{0}}=1-\frac{1}{2} \varepsilon(1-\sqrt{\xi}) \frac{\left[w_{0}^{\prime \prime}(0)\right]^{2}+\left[w_{0}^{\prime \prime}(1)\right]^{2}}{\int_{0}^{1}\left[w_{0}^{\prime \prime}(s)\right]^{2} \mathrm{~d} s}+O\left(\varepsilon^{2}\right),
$$

that, in the absence of rotary inertia, reduces to

$$
\frac{\omega}{\omega_{0}}=1-4 \varepsilon(1-\sqrt{\xi})+O\left(\varepsilon^{2}\right) .
$$

Fig.2 plots the approximated ratio (50) onto the numerical solution of the TPNM and shows that the 1-term correction provides excellent agreement for the fundamental mode. It is also clear from Eq.(50) that, as in the S-S situation, a perfectly reasonable limit is retrieved for the PNLM, i.e. for $\xi \rightarrow 0$.

The asymptotic expansion for the eigenmode reads

$$
w=w_{0}+\varepsilon w_{1}+O\left(\varepsilon^{2}\right)
$$


where $w_{0}$ and $w_{1}$ belong to the outer solution and they are given by (28), with coefficients (41), and by (47), respectively. We observe that the boundary layer terms are $O\left(\varepsilon^{2}\right)$ and therefore they do not appear explicitly in (51). To incorporate them consistently, one needs to consider the successive approximation term, $\varepsilon^{2} w_{2}$, for the outer solution.

\subsection{Beam with clamped and simply supported ends}

To fix ideas, let the left beam end be clamped and the right simply supported. The correspondent boundary conditions are given by (11a), (12b) and the pair of additional conditions (18). In this case, we arrive at $\gamma_{1}=2$ and $\gamma_{2}=3$ for the left and for the right boundary layer, respectively.

At leading order, one has the classical boundary conditions

$$
w_{0}(0)=w_{0}^{\prime}(0)=w_{0}(1)=w_{0}^{\prime \prime}(1)=0
$$

whence we get the constants in the general solution (28)

$$
\begin{aligned}
& c_{01}=-2 \lambda_{0}^{2}\left(\alpha^{2} \beta^{-2} \cosh \alpha+\cos \beta\right), \\
& c_{02}=2\left(\lambda_{0}^{2} \sin \beta+\alpha^{2} \sinh \alpha\right), \\
& c_{03}=-\lambda_{0}^{2} \sin \beta-\beta^{2} \cos \beta-\mathrm{e}^{\alpha} \alpha^{2}, \\
& c_{04}=\mathrm{e}^{\alpha}\left(\beta^{2} \cos \beta-\lambda_{0}^{2} \sin \beta\right)+\alpha^{2},
\end{aligned}
$$

together with Eq.(43a). The eigenvalues $\lambda_{0}=\lambda_{0}^{(n)}$ are found from the transcendental equation

$$
\alpha \cosh \alpha \sin \beta-\beta \cos \beta \sinh \alpha=0,
$$

that, when $\theta=0$, boils down to

$$
T\left(\lambda_{0}\right) U\left(\lambda_{0}\right)=S\left(\lambda_{0}\right) V\left(\lambda_{0}\right)
$$

266 The last equation amounts to the well known classical equation $\tanh \lambda_{0}=\tan \lambda_{0}$, while the correspondent eigenmodes are given by

$$
w_{0}(s)=C\left[U\left(\lambda_{0} s\right)-\frac{S\left(\lambda_{0}\right)}{T\left(\lambda_{0}\right)} V\left(\lambda_{0} s\right)\right] .
$$




$$
w_{1}(0)=0, \quad w_{1}^{\prime}(0)=(1-\sqrt{\xi}) w_{0}^{\prime \prime}(0), \quad w_{1}(1)=w_{1}^{\prime \prime}(1)=0,
$$

269

and $a_{10}$ and $a_{20}$ are defined by Eqs.(43a,32b)

$$
\begin{aligned}
& a_{10}=C \lambda_{0}^{2} \sqrt{\xi}(1-\sqrt{\xi}), \\
& a_{20}=C \lambda_{0}^{3} \sqrt{\xi}(1-\sqrt{\xi})\left[V\left(\lambda_{0}\right)-\frac{S^{2}\left(\lambda_{0}\right)}{T\left(\lambda_{0}\right)}\right] .
\end{aligned}
$$

The inhomogeneous equation (44), subject to the boundary conditions (54), possesses a solution provided that compatibility is satisfied, whereby we get the first eigenfrequency correction

$$
\lambda_{1}=-\lambda_{0} \frac{(1-\sqrt{\xi})\left[w_{0}^{\prime \prime}(0)\right]^{2}}{4 \int_{0}^{1}\left[w_{0}^{\prime \prime}(s)\right]^{2} \mathrm{~d} s} .
$$

The solution of the BVP $(44,54)$ has the form $(47)$ as for the C-C case, yet with different coefficients. Indeed, in the special case $\theta=0$, Eq.(56) simplifies to

$$
\lambda_{1}=-\lambda_{0}(1-\sqrt{\xi}),
$$

and the particular solution becomes

$$
w_{1 p}(s)=\frac{\lambda_{1}}{\lambda_{0}} s w_{0}^{\prime}(s)=C \lambda_{1} s\left[T\left(\lambda_{0} s\right)-\frac{S\left(\lambda_{0}\right)}{T\left(\lambda_{0}\right)} U\left(\lambda_{0} s\right)\right],
$$

whence

$$
\begin{aligned}
w_{1}(s) & =C \lambda_{0}(1-\sqrt{\xi})\left[T\left(\lambda_{0} s\right)-\frac{S\left(\lambda_{0}\right) U\left(\lambda_{0}\right)}{T\left(\lambda_{0}\right) V\left(\lambda_{0}\right)} V\left(\lambda_{0} s\right)\right]+w_{1 p}(s) \\
& =C \lambda_{0}(1-\sqrt{\xi})\left[(1-s) T\left(\lambda_{0} s\right)+\frac{S\left(\lambda_{0}\right)}{T\left(\lambda_{0}\right)}\left(s U\left(\lambda_{0} s\right)-\frac{U\left(\lambda_{0}\right)}{V\left(\lambda_{0}\right)} V\left(\lambda_{0} s\right)\right)\right] .
\end{aligned}
$$

25 Finally, we arrive at the following asymptotic expansion for the frequency ratio

$$
\frac{\omega}{\omega_{0}}=1-\frac{1}{2} \varepsilon(1-\sqrt{\xi}) \frac{\left[w_{0}^{\prime \prime}(0)\right]^{2}}{\int_{0}^{1}\left[w_{0}^{\prime \prime}(s)\right]^{2} \mathrm{~d} s}+O\left(\varepsilon^{2}\right)
$$

that, in the case $\theta=0$, reduces to

$$
\frac{\omega}{\omega_{0}}=1-2 \varepsilon(1-\sqrt{\xi})+O\left(\varepsilon^{2}\right) .
$$



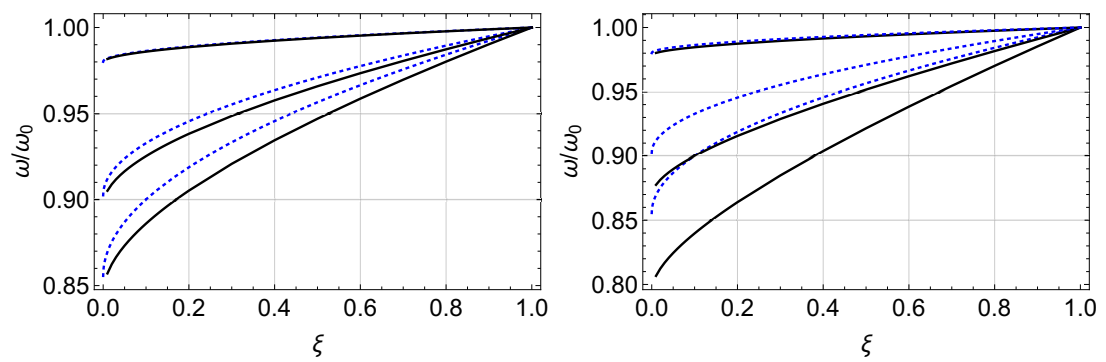

Figure 3: 1st (left) and 2nd (right) eigenfrequencies $\omega$ for a C-S beam (solid, black) in the absence of rotatory inertia, $\theta=0$, and with $\varepsilon=0.01,0.05$ and 0.075 , superposed onto the 1-term (dotted, blue) asymptotic approximation, normalized with respect to the classical local frequency $\omega_{0}$, Eq.(59)

Eq.(59) is plotted in Fig.3 alongside the numerical solution of the TPNM. Although the accuracy of the expansion is restricted to small values of $\varepsilon$, we still appreciate a limit as the TPNM tends to the PNLM.

\subsection{Cantilever Beam}

For a cantilever beam we have, at leading order,

$$
w_{0}(0)=w_{0}^{\prime}(0)=w_{0}^{\prime \prime}(1)=w_{0}^{\prime \prime \prime}(1)+\theta \lambda_{0}^{4} w_{0}^{\prime}(1)=0,
$$

and the constants in the general solution (28) are given by Eqs.(52), i.e. they are the same as in the $\mathrm{C}-\mathrm{S}$ case. The secular equation now reads

$$
\left(1+\frac{1}{2} \theta^{2} \lambda_{0}^{4}\right) \cosh \alpha \cos \beta-\frac{1}{2} \theta \lambda_{0}^{2} \sinh \alpha \sin \beta+1=0,
$$

that, in the special case of vanishing rotational inertia, reduces to

$$
S^{2}\left(\lambda_{0}\right)-T\left(\lambda_{0}\right) V\left(\lambda_{0}\right)=0 .
$$

This formula coincides with the classical result $\cosh \lambda_{0} \cos \lambda_{0}+1=0$ and the corresponding eigenforms are still given by Eq.(53).

In the first-order approximation, one arrives at the following boundary conditions

$$
\begin{aligned}
w_{1}(0) & =0, & w_{1}^{\prime}(0) & =(1-\sqrt{\xi}) w_{0}^{\prime \prime}(0), \\
w_{1}^{\prime \prime}(1) & =0, & w_{1}^{\prime \prime \prime}(1)+\lambda_{0}^{4} \theta w_{1}^{\prime}(1) & =-4 \lambda_{0}^{3} \lambda_{1} \theta w_{0}^{\prime}(1) .
\end{aligned}
$$



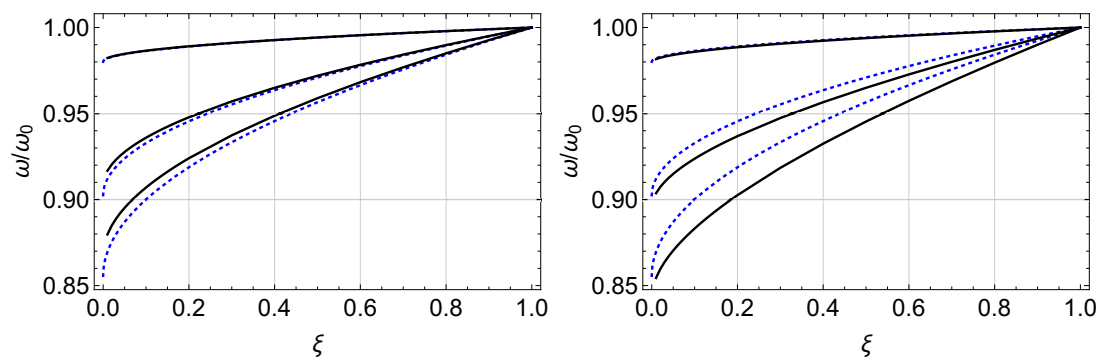

Figure 4: 1st (left) and 2nd (right) eigenfrequencies $\omega$ for a cantilever beam (solid, black) in the absence of rotatory inertia, $\theta=0$, and with $\varepsilon=0.01,0.05$ and 0.075 , superposed onto the 1-term (dotted, blue) asymptotic approximation, normalized with respect to the classical local model frequency $\omega_{0}$, according to Eq.(59)

together with the right boundary layer amplitude

$$
a_{20}=\sqrt{\xi}(1-\sqrt{\xi})\left[w_{1}^{\prime \prime}(1)+w_{0}^{\prime \prime \prime}(1)\right]
$$

the left being given by Eq.(43a). The compatibility condition for the inhomogeneous BVP $(44,60)$ is still given by Eq.(56) and, as a consequence, the ratio $\omega / \omega_{0}$ and the corresponding eigenmode correction are once again retrieved. Fig.4 compares the normalized eigenfrequency $\omega / \omega_{0}$ as numerically evaluated for the TPNM with the 1-term expansion (59) and shows good accuracy. Besides, the numerical solution curve matches the corresponding result given in Fig.5 of [10].

\section{Purely nonlocal model}

From the previous analysis, it clearly appears that the situation $\xi \rightarrow 0$ lends a perfectly admissible eigenfrequency which, therefore, can be assumed as the proper solution to the PNLM. We now consider what happens to the eigenmodes and for this we need to investigate the behavior of the boundary layer term $B_{\xi}(s)=\sqrt{\xi} \exp [-s /(\varepsilon \sqrt{\xi})], 0 \leq s \leq 1$, as $\xi \rightarrow 0$. Clearly, this is a transcendentally small term for $s>0$ and $B_{\xi}(s) \rightarrow 0$ uniformly. Non uniformity arises when we consider $s=0$ for then a boundary layer appears that may be studied taking the rescaled variable $s^{*}=s /(\varepsilon \sqrt{\xi})$, see [14]. This boundary layer 
is vanishingly small as $\xi \rightarrow 0$ but not so are its derivatives with respect to $s$

$$
B_{\xi}^{\prime}(s) \rightarrow\left\{\begin{array} { l l } 
{ 0 , } & { s > 0 , } \\
{ - \varepsilon ^ { - 1 } , } & { s = 0 , }
\end{array} \quad \text { and } \quad B _ { \xi } ^ { \prime \prime } ( s ) \rightarrow \left\{\begin{array}{ll}
0, & s \neq 0, \\
+\infty, & s=0,
\end{array}\right.\right.
$$

This result is the analogue of the steep boundary layer described in [37] under static axial deformation. We may now ask whether this unboundedness in the second derivative leads to an unbounded bending energy. To answer this we first observe that $\forall \eta>0, \int_{0}^{\eta} B_{\xi}^{\prime \prime}(s) \mathrm{d} s \rightarrow \varepsilon^{-1}$ uniformly and therefore $B_{\xi}^{\prime \prime}(s)$ is proportional to Dirac's delta function. Indeed, when considering the contribution $M_{\xi}$ of the boundary layer $B_{\xi}$ to the bending moment $M$ through Eq.(4), we find

$$
M_{\xi}(0) \rightarrow\left(2 \varepsilon^{2}\right)^{-1},
$$

at leading order. If we use this result in, say, the eigenmodes (38) for a S-S beam, we easily see that the boundary condition $M(0)=0$ is satisfied at leading order, for the boundary layer cancels out the contribution of the outer solution. At the same time, the constitutive BCs are asymptotically satisfied for a vanishingly small $\xi$ due to the asymptotic procedure applied above. We then conclude that, in the limit as $\xi \rightarrow 0$, the boundary layer warrants the fulfilment of all boundary conditions and it brings a finite contribution to the bending energy. From the standpoint of displacements, we get

$$
w(s) \rightarrow w^{(m)}+\varepsilon^{\gamma_{1}-1} a_{10} R(-s)+\varepsilon^{\gamma_{2}-1} a_{20} R(s-1),
$$

where $R(s)$ is the ramp function. For a S-S beam, we have $\gamma_{1}=\gamma_{2}=3$ and

$$
a_{10}=(-1)^{n+1} a_{20}=C \beta^{3} .
$$

Whence, a finite jump in the rotation and a concentrated couple at the beam ends is produced. This is perhaps not so surprising, for solutions in the sense of distributions are to be expected when an integral form of the constitutive equation is adopted. Consequently, from a mathematical standpoint, an energy bounded solution of the PNLM may be consistently defined as the limit of the TPNM, although it is meaningful in the sense of distributions and we may want to reject it on physical grounds. 


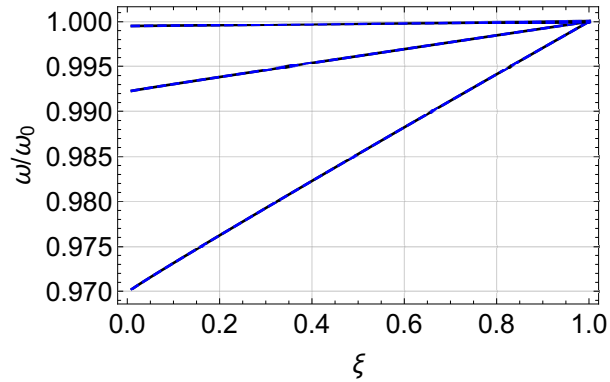

Figure 5: Eigenfrequency $\omega$ for modes 1, 2 and 4 for a S-S beam, normalized over the classical frequency $\omega_{0}$, for $\theta=0,1 / 100$ and $1 / 10$, as a function of the local model fraction $\xi$. As it occurs for the asymptotic expansion (39), the frequency ratio is unaffected by rotational inertia and curves overlap
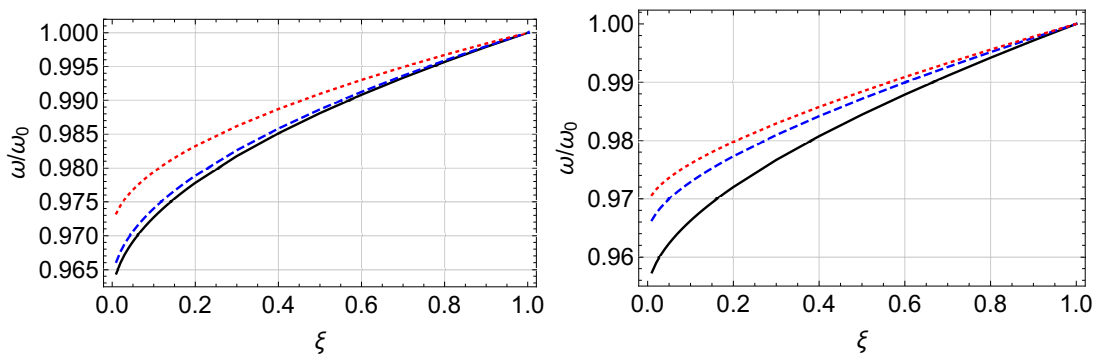

Figure 6: Eigenfrequency ratio $\omega / \omega_{0}$ for modes 1 (left panel) and 4 (right) for a C-C beam for $\theta=0$ (solid, black), $\theta=1 / 100$ (dashed, blue) and 1/10 (dotted, red), as a function of the local model fraction $\xi$

\section{Influence of rotational inertia}

We now consider the effect of including rotational inertia when considering the solution of the TPNM. Fig.5 plots the frequency ratio $\omega / \omega_{0}$ for mode numbers $n=1,4$ and 8 for a S-S beam and $\theta=0,1 / 100$ and 1/10. It appears that, for the S-S end conditions, rotational inertia is irrelevant for the purpose of determining the frequency ratio (yet it still affects $\omega_{0}$ ). Fig.6 plots the frequency ratio $\omega / \omega_{0}$ for mode numbers $n=1$ and 4 for $\theta=0,1 / 100$ and $1 / 10$ in a C-C beam. This time, rotational inertia plays an important role in the direction of contrasting the softening effect induced by the nonlocal fraction. Indeed, this hardening effect is already well manifest in the fundamental mode and, as ex- 

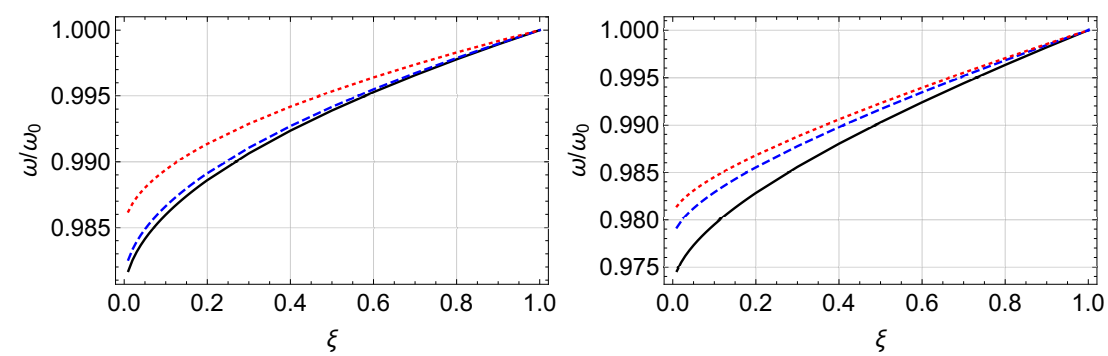

Figure 7: Eigenfrequency ratio $\omega / \omega_{0}$ for modes 1 (left panel) and 4 (right) for a C-S beam for $\theta=0$ (solid, black), $\theta=1 / 100$ (dashed, blue) and 1/10 (dotted, red), as a function of the local model fraction $\xi$
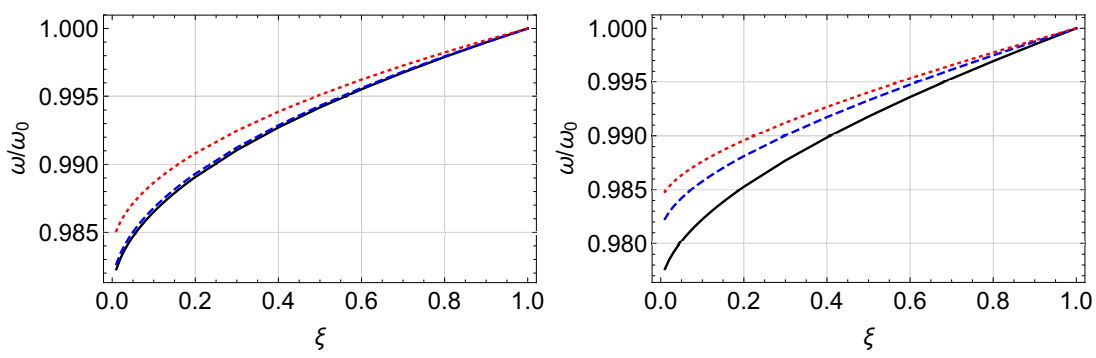

Figure 8: Eigenfrequency ratio $\omega / \omega_{0}$ for modes 1 (left panel) and 4 (right) for a C-F beam for $\theta=0$ (solid, black), $\theta=1 / 100$ (dashed, blue) and 1/10 (dotted, red), as a function of the local model fraction $\xi$ 
pected, it becomes stronger for higher modes. Besides, encompassing rotational inertia of the cross-section has a significant bearing on higher modes, regardless of the actual value of $\theta$. The same qualitative picture appears in Fig.7 and in Fig.8, respectively for $\mathrm{C}-\mathrm{S}$ and $\mathrm{C}-\mathrm{F}$ beams. It appears that the softening effect is stronger moving from $\mathrm{S}-\mathrm{S}$ to $\mathrm{C}-\mathrm{C}, \mathrm{C}-\mathrm{F}$ and then to $\mathrm{C}-\mathrm{S}$.

\section{Conclusions}

The purely nonlocal theory of elasticity has recently attracted considerable attention for the controversial results it conveys. Indeed, this model is believed to lead to ill-posed problems, owing to the appearance of a pair of constitutive boundary conditions which are generally at odd with the natural boundary conditions. In this paper, we approach the problem from a different perspective and carry out an asymptotic analysis of the free vibrations of flexural beams endowed with rotational inertia, within the two-phase theory of nonlocal elasticity. We show that the nonlocal term contributes with a boundary layer whose strength greatly varies for different end conditions. In the case of simply supported beams, the boundary layer is the weakest and we provide a two-term correction for the classical solution. Remarkably, this situation is affected by the presence of rotational inertia only in the classical sense. Conversely, clamped-clamped, clamped-supported and clamped-free (i.e. cantilever) conditions bring a much stronger boundary layer, a for these we provide a single correction term. Numerical results confirm the accuracy of the asymptotic approach and show that rotational inertia is very relevant in contrasting the softening effect connected to the nonlocal phase. Most interestingly, for any end condition, the asymptotic solution still exists and its energy remains bounded in the limit of the purely nonlocal theory, that is for a vanishingly small local phase. This is in contrast to what is anticipated in the literature, see, for instance, [30]. We are therefore in the position of attaching a meaning to the purely nonlocal theory, as the limit of the two-phase theory. In so doing, we encounter a solution that is defined in the sense of distributions (for the curvature) and, although maybe questionable 
from a physical standpoint, it is mathematically sound.

\section{Acknowledgments}

GM gratefully acknowledges a Visiting Professor Position granted by the University of Modena and Reggio Emilia in the AY 2018/2019.

AN welcomes support from FAR2019, Piano di sviluppo dipartimentale DIEF, DR nr. 498/2019.

\section{References}

[1] N Challamel and CM Wang. The small length scale effect for a non-local cantilever beam: a paradox solved. Nanotechnology, 19(34):345703, 2008.

[2] E Cosserat and F Cosserat. Théorie des corps déformables. Herman et Flis, Paris, 1909.

[3] T M Dai. Renewal of basic laws and principles for polar continuum theories (i) micropolar continua. Applied Mathematics and Mechanics, 24:1119$1125,2003$.

[4] MA Eltaher, ME Khater, and S A Emam. A review on nonlocal elastic models for bending, buckling, vibrations, and wave propagation of nanoscale beams. Applied Mathematical Modelling, 40(5-6):4109-4128, 2016.

[5] A C Eringen. Linear theory of nonlocal elasticity and dispersion of plane waves. International Journal of Engineering Science, 10(5):425-435, 1972.

[6] A C Eringen. On differential equations of nonlocal elasticity and solutions of screw dislocation and surface waves. Journal of applied physics, 54(9): 4703-4710, 1983.

[7] A C Eringen. Theory of nonlocal elasticity and some applications. Technical report, PRINCETON UNIV NJ DEPT OF CIVIL ENGINEERING, 1984. 
[8] A C Eringen. Nonlocal continuum field theories. Springer Science \& Business Media, 2002.

[9] J. Fernández-Sáez and R. Zaera. Vibrations of bernoulli-euler beams using the two-phase nonlocal elasticity theory. International Journal of Engineering Science, 119:232-248, 2017.

[10] J Fernández-Sáez, R Zaera, JA Loya, and JN Reddy. Bending of eulerbernoulli beams using eringen's integral formulation: a paradox resolved. International Journal of Engineering Science, 99:107-116, 2016.

[11] AL Gol'denveizer. Theory of Thin Elastic Shells. International Series of Monograph in Aeronautics and Astronautics. Pergamon Press, New York, 1961.

[12] J Kaplunov and A Nobili. A robust approach for analysing dispersion of elastic waves in an orthotropic cylindrical shell. Journal of Sound and Vibration, 401:23-35, 2017.

[13] I A Karnovsky and O Lebed. Advanced methods of structural analysis. Springer Science \& Business Media, 2010.

[14] J Kevorkian and J D Cole. Multiple scale and singular perturbation methods, volume 114 of Applied Mathematical Sciences. Springer-Verlag New York, Inc, 1996.

[15] C C Koutsoumaris and KG Eptaimeros. A research into bi-Helmholtz type of nonlocal elasticity and a direct approach to Eringen's nonlocal integral model in a finite body. Acta Mechanica, 229(9):3629-3649, 2018.

[16] P Lu, HP Lee, C Lu, and PQ Zhang. Dynamic properties of flexural beams using a nonlocal elasticity model. Journal of applied physics, 99(7):073510, 2006.

[17] G A Maugin. A historical perspective of generalized continuum mechanics. In Mechanics of Generalized Continua, pages 3-19. Springer, 2011. 
[18] L Meng, D Zou, H Lai, Z Guo, X He, Z Xie, and C Gao. Semi-analytic solution of Eringen's two-phase local/nonlocal model for Euler-Bernoulli beam with axial force. Applied Mathematics and Mechanics, 39(12):18051824, 2018.

[19] G. Mikhasev, E. Avdeichik, and D. Prikazchikov. Free vibrations of nonlocally elastic rods. Mathematics and Mechanics of Solids, pages 888-896, 2018. doi: $10.1177 / 1081286518785942$.

[20] GI Mikhasev. On localized modes of free vibrations of single-walled carbon nanotubes embedded in nonhomogeneous elastic medium. ZAMM, 94(1-2): 130-141, 2014.

[21] GI Mikhasev and MG Botogova. Free localized vibrations of a long doublewalled carbon nanotube introduced into an inhomogeneous elastic medium. Vestnik St. Petersburg University: Mathematics, 49(1):85-91, 2016.

[22] A Nobili and L Lanzoni. Electromechanical instability in layered materials. Mechanics of Materials, 42(5):581 - 591, 2010. ISSN 0167-6636. doi: https: //doi.org/10.1016/j.mechmat.2010.02.006.

[23] A Nobili, E Radi, and A Vellender. Diffraction of antiplane shear waves and stress concentration in a cracked couple stress elastic material with micro inertia. Journal of the Mechanics and Physics of Solids, 124:663-680, 2019.

[24] J Peddieson, R Buchanan, and RP McNitt. Application of nonlocal continuum models to nanotechnology. International Journal of Engineering Science, 41(3):305-312, 2003.

[25] W Pietraszkiewicz and VA Eremeyev. On natural strain measures of the non-linear micropolar continuum. International Journal of Solids and Structures, 46:774-787, 2009.

[26] A A Pisano and P Fuschi. Closed form solution for a nonlocal elastic bar in tension. International Journal of Solids and Structures, 40(1):13-23, 2003. 
[27] A. Polyanin and A. Manzhirov. Handbook of Integral Equations. CRC Press, New York, 2008.

[28] A D Polyanin and A V Manzhirov. Handbook of integral equations. CRC press, 1998.

[29] H Rafii-Tabar, E Ghavanloo, and S A Fazelzadeh. Nonlocal continuumbased modeling of mechanical characteristics of nanoscopic structures. Physics Reports, 638:1-97, 2016.

[30] G Romano, R Barretta, M Diaco, and F M de Sciarra. Constitutive boundary conditions and paradoxes in nonlocal elastic nanobeams. International Journal of Mechanical Sciences, 121:151-156, 2017.

[31] V I Smirnov. A course of higher mathematics, volume 1. Pergamon, 1964.

[32] Q Wang and KM Liew. Application of nonlocal continuum models to nanotechnology. Phys Lett A, 363(3):236-242, 2007.

[33] Q. Wang and V.K. Varadan. Wave characteristics of carbon nanotubes. International Journal of Solids and Structures, 43(2):254 - 265, 2006.

[34] YB Wang, XW Zhu, and HH Dai. Exact solutions for the static bending of Euler-Bernoulli beams using Eringen's two-phase local/nonlocal model. AIP Advances, 6(8):085114, 2016.

[35] F Yang, ACM Chong, DCC Lam, and P Tong. Couple stress based strain gradient theory for elasticity. International Journal of Solids and Structures, 39(10):2731-2743, 2002.

[36] X Zhu, Y Wang, and H-H Dai. Buckling analysis of Euler-Bernoulli beams using Eringen's two-phase nonlocal model. International Journal of Engineering Science, 116:130-140, 2017.

[37] XiaoWu Zhu and HuiHui Dai. Solution for a nonlocal elastic bar in tension. Science China Physics, Mechanics and Astronomy, 55(6):1059-1065, 2012. 